\title{
Some Properties of a Connected Topological Group
}

\author{
Beshimov R. B. ", Zhuraev R. M. \\ National University of Uzbekistan named after Mirzo Ulugbek, Uzbekistan
}

Copyright@2019 by authors, all rights reserved. Authors agree that this article remains permanently open access under the terms of the Creative Commons Attribution License 4.0 International License

\begin{abstract}
In this paper, we study some topological properties of connected topological groups. From a logical point of view, the concept of a topological group arises as a simple combination of the concepts of a group and a topological space. In the same set $G$, operations multiplication and topological closure are specified simultaneously.
\end{abstract}

Keywords Subgroup, Coset, Dense Set, Connectedness, Hereditary Disconnected, Extremally Disconnected, Locally Connected

\section{Introduction}

Definition 1.1 [2]. A topological group is the set $G$ on which both topological and group structures are given. This requires that the maps

$$
G \times G \rightarrow G:(x ; y) \mapsto x \circ y
$$

and

$$
G \rightarrow G: x \mapsto x^{-1} \text { be continuous. }
$$

A topological group (or shortly $G$ - group) is a topological space that is simultaneously a group, with $x y$ being a continuous function of $x$ and $y$ and $x^{-1}$ being a continuous function of $x$. In this case, the following are added to the four axioms of the group and the two axioms of open sets:

TG1. For each neighborhood $U(a b)$ of the product $a b$ there are neighborhoods $V(a)$ and $W(b)$ whose product is contained in $U(a b)$.

TG2. For each neighborhood $U\left(a^{-1}\right)$, there exists a neighborhood $V(a)$ such that $V(a)^{-1}$ is contained in $U\left(a^{-1}\right)$.

From TG1 and TG2, the statements readily follow:

TG1/1. For each neighborhood $U\left(a^{-1} b\right)$ there are neighborhoods $V(a)$ and $W(b)$ such that
$V(a)^{-1} W(b)$ is contained in $U\left(a^{-1} b\right)$.

TG2/1. For each neighborhood $U\left(a b^{-1}\right)$ there are neighborhoods $\quad V(a)$ and $W(b)$ such that $V(a) W(b)^{-1}$ is contained in $U\left(a b^{-1}\right)[2]$.

The topological group is usually denoted by $\langle G, \theta, 0\rangle$.

Suppose $F$ is a closed set, $U$ is an open set, $P$ is an arbitrary set, and $a$ is some element of the topological group $G$. Then $F a, a F, F^{-1}$ are closed sets; $U P$, $P U, U^{-1}$ are open sets [6].

If a basis at the unit $e$ is given, then all neighborhoods of this element are specified: those will be the sets $U(e)$, which contain at least one of the basis neighborhoods. But then the neighborhoods and other points are known, because if $U(e)$ is a neighborhood of the unit $e$, then $a U(e)$ is a neighborhood of $a$ and all neighborhoods of $a$ can be represented in this form[6].

Indeed, if $U(a)$ is an arbitrary neighborhood of $a$, then $a^{-1} U(a)$ is an open set and $e \in a^{-1} U(a)$, then $U(e)=a^{-1} U(a)$, therefore, we have $U(a)=a U(e)$ One can call $a U(e)$ «neighborhood of the point $e$ shifted by $a \gg[4]$.

The sets of all neighborhoods of $e \in G$ are denoted by $\Omega$.

The following properties are true [3]:

1. $e \in U$ for all $U \in \Omega$.

2. if $U_{1}, U_{2} \in \Omega$, then exists $U \in \Omega$ since $U \subset U_{1} \cap U_{2}$

3. if $U$ is open subset and $a \in U$, then exists $V \in \Omega$ since $a V \subset U$

4. if $U \in \Omega$ and $a \in G$, then exists $V \in \Omega$ since $a \mathrm{Va}^{-1} \subset U$

5. if $U \in \Omega$, then exists $V \in \Omega$ since $V V^{-1} \subset U$

The closure of $A$ is denoted by $\bar{A}$. 


\section{Proposition 1.1 [5]}

Let $G$ be a topological group and $E \subset G$ be an arbitrary subset of $G$, then $\bar{E}=\bigcap_{U \in \Omega} U E=\bigcap_{U \in \Omega} E U$.

\section{Proposition 1.2 [4]}

Let $G$ be a group and $g \in G, M_{\alpha}$ a family a subset of $G$ for each $\alpha \in I$. Then $g\left(\bigcap_{\alpha \in I} M_{\alpha}\right)=\bigcap_{\alpha \in I} g M_{\alpha}$, where $I$ is the set of indices.

We introduce the following definition and the corresponding theorems.

\section{Definition 1.2 [4]}

Let $(G, *)$ be a group and $H$ be a non-empty subset of $G$. Then $(H, *)$ is called a subgroup of $(G, *)$, if $(H, *)$ is a group.

\section{Definition 1.3 [4]}

Let $(G, *)$ be a group. A subset $H$ of a group $G$ is called a normal subgroup of $G$, if $a H=H a$ for all $a \in G$.

\section{Definition 1.4 [4]}

Let $H$ be a subgroup of $G$. Then the number of distinct left cosets (written $[G: H]$ ) of $H$ is called the index of $H$ in $G$.

A topological space $X$ is called connected [2], if $X$ cannot be decomposed into a union of non-empty open non-intersecting sub sets.

\section{Proposition 1.3 [2]}

A topological space $X$ is connected if and only if $X$ does not have its proper subset $Y$ (i.e. $Y \neq \varnothing, X$ ), which is closed and open simultaneously.

A connected component [2] of a topological space $X$ is any maximal connected subset in $X$.

If $x_{0} \in X$ is a fixed point, then the union $K\left(x_{0}\right)=\cup M_{x_{0}}$ of all connected subsets of $M_{x_{0}} \subset X$ containing $x_{0}$ is connected. It is easy to show that $K\left(x_{0}\right)$ is not a proper subset of any other connected set, i.e. $K\left(x_{0}\right)$ connected component containing the point $x_{0}$.

Theorem 1.1 [7]

Let $A, B \subseteq G$ be arbitrary non-empty subsets in a topological group $G$ and $b \in G$ an arbitrary element of $G$. Then the following statements are true:

1) If $A$ is a connected set in a topological group $G$, then $A^{-1}$ is a connected set in a topological group $G$.

2) If a $A$ connected set in the topological group $G$, then $b A$ and $A b$ are connected sets in the topological group $G$.

3) If $A$ and $B$ are non-empty connected sets in the topological group $G$, then the set $A \cdot B$ is a connected set in the topological group $G$.

\section{Theorem1.2 [7]}

If $G$ is a topological group and $C_{e}$ is a connected component of the unit $e$ in the topological group $G$, then it is a closed normal subgroup in the topological group $G$, and for any element $g \in G$ the set $C_{e} g$ is a connected component of the point $g$ in the topological group $G$ and $C_{e} g=g C_{e}$.

\section{Main Results}

\section{Proposition 2.1}

Let $G$ be a topological group and $E \subset G$ be an arbitrary subset, $a \in G$, then

a. $\overline{a E}=a \bar{E}(\overline{E a}=\bar{E} a)$.

b. $\operatorname{Int}(a E)=a \operatorname{Int}(E)(\operatorname{Int}(E a)=\operatorname{Int}(E) a)$.

Proof: a) By proposition 1.1 we have $\overline{a E}=\bigcap_{U \in \Omega}(a E) U \quad\left(\overline{E a}=\bigcap_{U \in \Omega} U(E a)\right) \quad . \quad$ By associatively of the group operation $(a E) U=a(E U)(U(E a)=(U E) a)$ and we have $\bigcap_{U \in \Omega}(a E) U=\bigcap_{U \in \Omega} a(E U)$

$\left(\bigcap_{U \in \Omega} U(E a)=\bigcap_{U \in \Omega}(U E) a\right)$. Now, by virtue of proposition 1.2, we have $\bigcap_{U \in \Omega} a(E U)=a \bigcap_{U \in \Omega} E U$ $\left(\bigcap_{U \in \Omega}(U E) a=\left(\bigcap_{U \in \Omega} U E\right) a\right) \quad, \quad$ then $\quad$ the equality $\overline{a E}=a \bar{E}(\overline{E a}=\bar{E} a)$ holds.

b) Firstly, we prove that a map $L_{a}: G \rightarrow G$ is homeomorphism, at $L_{a}(x)=a x$ for all $x \in G$ (where $a$ is a fixed element of group $G$ ). The mapping $L_{a}$ is one-to-one. Indeed, for every element $y^{\prime}$ there is an element $x^{\prime}$, and moreover, there is only one such that 
$y^{\prime}=a x^{\prime}$. Further, the mapping $L_{a}$ is continuous. Indeed, if $y^{\prime}=a x^{\prime}$ and $W$ are some neighborhood of the element $y^{\prime}$, then there exist neighborhoods $U$ and $V$ of the elements $a$ and $x^{\prime}$ such that $U V \subset W$; but $a \in U$ and, therefore, $a V \subset W$, i.e. $L_{a}(V) \subset W$, which means the continuity of the mapping $L_{a}$.The continuity of the inverse mapping $L_{a}^{-1}(y)=a^{-1} y$ is also proved. Then by the homeomorphism property we have that

$$
\operatorname{Int} L_{a}(E)=L_{a}(\operatorname{Int} E)
$$

.Hence,

$\operatorname{Int}(a E)=\operatorname{aInt}(E)$.Secondly, a map $R_{a}: G \rightarrow G$ (at $R_{a}(x)=x a$ for all $x \in G$ ) is a homeomorphism. Because, the $R_{a}$ mapping is one-to-one. Indeed, for every element $y^{\prime}$ there is an element $x^{\prime}$, and moreover, there is only one such that $y^{\prime}=x^{\prime} a$. Further, the mapping $R_{a}$ is continuous. Indeed, if $y^{\prime}=x^{\prime} a$ and $W$ are some neighborhood of the element $y^{\prime}$, then there exist neighborhoods $U$ and $V$ of the elements $x^{\prime}$ and $a$ such that $U V \subset W$; but $a \in V$ and, therefore, $U a \subset W$, i.e. $R_{a}(V) \subset W$, which means the continuity of the mapping $R_{a}$.The continuity of the inverse mapping $R_{a}^{-1}(y)=y a^{-1}$ is also proved. Then by the homeomorphism property we have that $\operatorname{Int}_{a}(E)=R_{a}(\operatorname{Int} E)$ for an arbitrary subset of $E \subset G$. Hence, $\operatorname{Int}(E a)=\operatorname{Int}(E) a$. Proposition 2.1 is completely proved.

\section{Theorem 2.1}

Let the topological group $G$ be connected:

1). then there is no open proper subgroup of group $G$.

2). if the subgroup $H$ has a finite index in the topological group $G$, then the subgroup $H$ is dense in $G$.

3). if subgroup $H$ has a finite index in the topological group $G$, then each cosets of group $G$ over subgroup $H$ is dense in $G$.

Proof. 1) Suppose the opposite. Let a topological group $G$ be connected and there is an open proper subgroup $H$ of the group $G$. We show that $H=\bar{H}$. It is known that $H \subset \bar{H}$ for arbitrary subset $H \subset G$. Choose an arbitrary point $a \in \bar{H}$. Then $a H$ will be an open subset in the topological space $G$ containing point $a$, and we have $a H \cap H \neq \varnothing$. Assume that $b \in a H$ and $b \in H$, then there exists $h \in H$ such that $b=a h \in H$. We have $a \in H h^{-1} \subset H H^{-1}=H$. In this way, $\bar{H}=H$. This means that the subgroup $H$ is clopen. By proposition 1.3 topological group $G$ is disconnected. This contradicts the condition of theorem 2.1. So, there is an open proper subgroup of the group $G$.

2) Now suppose that $H$ is a subgroup with a finite index of a topological group $G$. Then there exists a finite sequence $a_{1}, a_{2}, \ldots, a_{n}\left(a_{i} H \neq a_{j} H\right.$ for all $i \neq j$, $i=1,2, \ldots, n$ and $j=1,2, \ldots, n)$ of $G$, such that $a_{1} H \cup a_{2} H \cup \ldots \cup a_{n} H=G$. It is known that, $a_{i} H \subset a_{i} \bar{H} \quad$ for $\quad$ all $i=1,2, \ldots, n, \quad$ then $G=\left(a_{1} H \cup a_{2} H \cup \ldots \cup a_{n} H\right) \subset\left(a_{1} \bar{H} \cup a_{2} \bar{H} \cup \ldots \cup a_{n} \bar{H}\right) \subset G$ . Hence have, that $a_{1} \bar{H} \cup a_{2} \bar{H} \cup \ldots \cup a_{n} \bar{H}=G$. Now we prove that $\bar{H}$ is a subgroup of a topological group $G$. Let $g_{1}, g_{2} \in \bar{H}$ and $U$ is be an arbitrary neighborhood of point $g_{1} g_{2}$. Since we have continuity of the group operation with respect to both variables, there exist neighborhoods $V_{1}, V_{2}$ of points $g_{1}$ and $g_{2}$, respectively, such that $V_{1} V_{2} \subset U$. From the definition of closure, we get $g_{1} \in V_{1} \cap H$ and $g_{2} \in V_{2} \cap H$. This means that $g_{1} \in H, g_{2} \in H$. We get that $g_{1} g_{2} \in V_{1} V_{2}$ and since $H$ is a group we have $g_{1} g_{2} \in H$. Since $V_{1} V_{2} \subset U$ we get $g_{1} g_{2} \in U$, so $g_{1} g_{2} \in U \cap H$. Finally, we proved that $g_{1} g_{2} \in \bar{H}$. Let $h \in \bar{H}$ and $U$ is be an arbitrary neighborhood of a point $h^{-1}$. From the continuity of the inverse map we get $U^{-1}$ is neighborhood of a point $h$.From the definition of closure, we get $U^{-1} \cap H \neq \varnothing$. So, there exists point $g$ : such that $g \in U^{-1}$ and $g \in H$,then $g^{-1} \in H$ since $H$ is a subgroup of $G$. We get that $g^{-1} \in U$ and $g^{-1} \in H$, so $g^{-1} \in U \cap H$. In order to show $h^{-1} \in \bar{H}$ we need to prove $U \cap H \neq \varnothing$ for all $U$ is an arbitrary neighborhood of a point $h^{-1}$. It follows that $h^{-1} \in \bar{H}$, as required. Then we have that $\bar{H}$ is a closed subgroup with a finite index in the topological group $G$. Then coset $a \bar{H}$ is also closed in $G$. The union of all finite cosets, except of $\bar{H}$, is closed again. This union is a 
complement of the subgroup $\bar{H}$. Consequently, $\bar{H}$ is open. Hence, $\bar{H}$ is clopen subgroup in $G$. Then the proposition 1.3, we have that $\bar{H}=G$. Then $H$ is dense in $G$.

3) Suppose that $H$ subgroup has finite index connected of topological group $G$. Then by virtue of theorem 2.1 part 2), $H$ is dense in $G$, i.e. $\bar{H}=G$. By proposition 2.1 part a) we have that $\overline{x H}=x \bar{H}=x G=G(\overline{H x}=\bar{H} x=G x=G)$ for all $x \in G$. Then $x H(H x)$ is everywhere dense in $G$. Theorem 2.1 is completely proved.

For a subset $A$ of a group $G$ we write $A^{n}=\left\{a^{n}: a \in A\right\}$ [4].

\section{Definition 2.1[4]}

A group $G$ is called nilpotent if $G^{n}=\{e\}$ for some positive integer $n$.

\section{Proposition 2.2}

If $G$ is a Hausdorff topological group and $H$ is a nilpotent subgroup of a group $G$, then $\bar{H}$ also be a nilpotent subgroup of a group $G$.

Proof: According to theorem 2.1, $\bar{H}$ is a subgroup of a group $G$.

We prove that $\bar{H}$ is a nilpotent subgroup.

Assume that $H^{n}=\{e\}$. It means that $h^{n}=e$ for all $h \in H$. Suppose the opposite, and let $a \in \bar{H}$ such elements that $a^{n} \neq e$. Since $G$ is a Hausdorff topological group, then in a topological group $G$ there are such neighborhoods $U$ and $V$ elements $a^{n}$ and $e$, respectively, that $U \cap V=\varnothing$.

It follows from the continuity of the multiplication operation that there exist such neighborhoods $U_{1}(a), U_{2}(a), \ldots, U_{n}(a)$ of $a$ such that $U_{1}(a) U_{2}(a) \ldots U_{n}(a) \subset U$. It is known that $U(a)=\bigcap_{i=1}^{n} U_{i}(a)$ is a neighborhood of $a$. It means that for all $j=1,2, \ldots, n$. In this case, we have $U(a) \cdot U(a) \cdot \ldots \cdot U(a) \subset U$ i.e. $U(a)^{n} \subset U$.

Since, $\quad a \in \bar{H}$ then $U(a) \cap H \neq \varnothing$. If $b \in U(a) \cap H$, then from the nilpotent subgroup $H$ it follows that $b^{n}=e$. Moreover $e=a^{n} \in U(a)^{n} \subset U$, so $U \cap V \neq \varnothing$.

We got a contradiction with the choice of neighborhoods
$V$ and $U$.

Denote by $C_{a}$ connected component of the point $a$ in the topological group.

The connected component of the neutral element $e$ of group $G$ is called the neutral component of this group [3].

\section{Theorem 2.2}

If $G$ is a topological group and $a, b \in G$ is an arbitrary element in $G$. Then the following statements are true:
1. $C_{a}=b \cdot C_{b^{-1} a}$
2. $C_{a b}=C_{a} \cdot C_{b}$
3. $C_{a^{-1}}=C_{a}^{-1}$

Proof. 1) First we show that $C_{a}=b C_{b^{-1} a}$. According to theorem 1.1, part 2), $b^{-1} C_{a}$ will be a connected set in the topological space $G$ containing point $b^{-1} a$. Then $b^{-1} \cdot C_{a} \subset C_{b^{-1} a}$ since $C_{a} \subset b \cdot C_{b^{-1} a}$. Similarly, $b \cdot C_{b^{-1} a}$ will be a connected set in the topological space $G$ containing the point $a$.Then $b \cdot C_{b^{-1} a} \subset C_{a}$.We have that $C_{a}=b C_{b^{-1} a}$

2) Now we show that $C_{a b}=C_{a} \cdot C_{b}$ By theorem 1.2, $C_{a b}=a b C_{e}=a b\left(C_{e} C_{e}\right)=a\left(b C_{e}\right) C_{e}$. We have that $C_{a b}=a\left(C_{e} b\right) C_{e}=\left(a C_{e}\right)\left(b C_{e}\right)=C_{a} C_{b}$.

3) By virtue of theorem 1.2, $C_{a^{-1}}=a^{-1} C_{e}$. It is known that $C_{e}$ is a normal subgroup of the topological group $G$. Then $C_{e}=C_{e}^{-1} \quad$ So $C_{a^{-1}}=a^{-1} C_{e}=a^{-1} C_{e}^{-1}=\left(C_{e} a\right)^{-1}=C_{a}^{-1}$. Theorem 2.2 is completely proved.

A topological space $X$ is called hereditarily disconnected [1] if $X$ does not contain any connected subsets of cardinality larger than one. Hence, a space $X$ is hereditarily disconnected if and only if the component of any point $x \in X$ consists of the point $x$ alone. Since the components of a space are closed, every hereditarily disconnected space is a $T_{1}$-space.

A topological space $X$ is called extremally disconnected [1], if $X$ is a Hausdorff space and for every open set $U \subset X$ the closure $\bar{U}$ is open in $X$. 
We say that a topological space $X$ is locally connected [1], if for every point $x \in X$ and any neighborhood $U$ of the point $x$ there exists a connected set $C \subset U$ such that $x \in \operatorname{Int} C$

\section{Theorem 2.3}

Let $G$ be a topological group. Then

a. $G$ is hereditary disconnected if and only if there is a connected component of a point $x \in G$ consisting only of $x$.

b. $G$ is extremally disconnected if and only if there exists an open set $U \subset G$ whose closure $[U]$ is open in $G$.

c. $\quad G$ is locally connected, if and only if for unit point $e \in G$ and any neighborhood $U$ of $\boldsymbol{e}$ there is a connected set $C \subset U$ such that $e \in \operatorname{Int} C$.

Proof: a) 1) It is clear that a topological group $G$ is hereditarily disconnected, then the component of every point $x \in G$ consists only of $x$.

2) Let $C_{x}=\{x\}$, where is $C_{x}$ is be a connected component of the point $x$ of the topological group $\mathrm{G}$. We show that $C_{a}=\{a\}$ for all $a \in G$, where $C_{a}$ is connected component of a point $a$. Then, by theorem 1.2, $x C_{e}=C_{x}$ since, $x C_{e}=\{x\}$. This equality of the product of both parts $a x^{-1} \in G$, we get $a C_{e}=\{a\}$. Now, still using theorems 1.2, it follows that $a C_{e}=C_{a}$. From where we have that $C_{a}=\{a\}$.

b) 1) It is known that if a topological group $G$ is extremally disconnected, then for every open set $U \subset X$ the closure $\bar{U}$ is open at $G$.

2) Suppose that there is an open $\operatorname{set} U \subset G$, such that the closure $\bar{U}$ is open in $G$. We choose point $x \in U$, then there exists a neighborhood $U_{e}$ of a point of a neutral element $e \in G$ such that $U=x U_{e}$. Then, by part a) of proposition 2.1 the closure $[U]=x\left[U_{e}\right]$ is open in $G$. Consequently, the closure $\left[U_{e}\right]$ is open at $G$. Choose arbitrary point $y \in G$. Then the set $y\left[U_{e}\right]=\left[y U_{e}\right]$ is open at $G$. Then for every open set $U_{y} \subset G$ the closure $\left[U_{y}\right]$ is open in $G$, where $U_{y}$ is a neighborhood of an arbitrary point $y \in G$. Therefore, the topological group $G$ is extremally disconnected.

c) 1) It is known that if a topological group $G$ is locally connected, then for each point $x \in G$ in particular $e \in G$ and any its neighborhood $U$ there exists a connected set $C \subset U$ such that $e \in \operatorname{Int} C$.
2) Choose an arbitrary point $x \in G$ topological group $G$. Let for a single point $e \in G$ and any its neighborhood $U$ there exists a connected set $C \subset U$, such that $e \in \operatorname{Int} C$. Then, any of its neighborhoods $U^{\prime}$ of a point $x$, there exists a neighborhood $U$ of a point $e \in G$ such that $U^{\prime}=x U$ and $x C \subset x U=U^{\prime}$. It is easy to see that the set $C^{\prime}=x C$ are connected. By virtue we get $x e \in x \operatorname{Int} C$. By proposition 2.1 part b) implies that $x \in \operatorname{Int}(x C)$. Then for each point $x \in G$ and any its neighborhood $U^{\prime}$ there exists a connected $\operatorname{set} C^{\prime} \subset U^{\prime}$, such that $x \in \operatorname{Int} C^{\prime}$. Therefore, a topological group $G$ is locally connected. Theorem 2.3 is completely proved.

\section{REFERENCES}

[1] R.Engelking, General Topology. Revised and completed edition. Berlin: Helderman, 1986, 752 p.

[2] A. V. Arkhangel' skii, "Classes of topological groups" AMS, 36: 3(1981), 127-146.

[3] N. Burbaki, General topology, topological groups of numbers and associated groups and spaces. Moscow 1969, $392 \mathrm{p}$.

[4] D. S. Malik, John N. Mordeson, M. K. Sen. Fundamentals of abstract algebra.1997, $636 \mathrm{p}$.

[5] A. V. Arkhangel' skii, «Topological homogeneity. Topological groups and their continuous images», Russian Math. Surveys,42:2(1987),83-131

[6] L. S. Pontryagin. Continuous groups. Moscow 1954, 516 p.

[7] V.I. Arnautov, G. N. Ermakov. Introduction to the theory of topological groups. Moldova. 2013, $215 \mathrm{p}$. 